\title{
Mobilization of an anomalous retro aortic coronary artery to prevent myocardial ischemia after aortic valve replacement
}

\author{
José Máximo $^{1}$, Paulo Pinho ${ }^{1}$, and Jorge Casanova ${ }^{1}$ \\ ${ }^{1}$ Centro Hospitalar Universitário de São João
}

March 21, 2021

\begin{abstract}
During valvular surgery, abnormal coronary distribution, especially if unidentified by preoperative exams, may put coronary arteries at risk of damage. In this case report, we describe the approach to an anomalous right coronary artery during aortic valve replacement.
\end{abstract}

Mobilization of an anomalous retro aortic coronary artery to prevent myocardial ischemia after aortic valve replacement

José Máximo $^{1,2}$, Paulo Pinho ${ }^{1,2}$, Jorge Casanova ${ }^{1,2}$

Cardiothoracic Surgery Center, Centro Hospitalar Universitário S. João, Porto, Portugal

Department of Surgery and Physiology, Faculty of Medicine, Porto University, Portugal

\section{Abstract}

During valvular surgery, abnormal coronary distribution, especially if unidentified by preoperative exams, may put coronary arteries at risk of damage. In this case report, we describe the approach to an anomalous right coronary artery during aortic valve replacement.

\section{Keywords}

Aortic valve replacement; Coronary origin and distribution anomalies; Anatomic variants; Preoperative study.

Word count

990 (Title, Abstract, Case Report, Discussion, Conclusion, References, Legends)

\section{Integrity statement}

- The authors have no conflicts of interest to declare and no external funding sources were used for manuscript writing.

- Informed consent for publishing data from clinical records and intraoperative images was obtained from the patient in written form.

- The main text, associated media and legends and patient written consent were assessed by the Ethics Committee of Centro Hospitalar de São João and Faculty of Medicine of Porto University, resulting in a statement of approval (included as "Supplementary File for Review").

\section{Corresponding author}

José Carlos Máximo Duarte

Centro Hospitalar S. João, Porto, Portugal 
Alameda Hernâni Monteiro, s/n, 4200-319, Porto, Portugal

\section{Case Report}

A 73-year-old male with the diagnosis of severe aortic stenosis was admitted in our cardiac surgery center for aortic valve replacement. He was otherwise healthy and his preoperative study, consistent with severe aortic stenosis, further revealed an anomalous origin of the left circumflex artery. As revealed in the coronariogram (Figure 1), the circumflex artery originated in the right coronary sinus, adjacent to the right coronary artery origin, and continued with a retro aortic trajectory, at the level of the aortic valve leaflets, until joining its most usual course in the atrioventricular groove. A thoracic computed tomography angiogram scan confirmed the finding (Figure 2).

After sternotomy, heparinization and mild hypothermic cardiopulmonary bypass (CPB) institution, a transverse aortotomy was performed, followed by an extensive periaortic dissection, to ensure the individualization of the anomalous coronary artery. The bicuspid aortic valve was considerably calcified, with fusion of the right coronary and non-coronary coronary sinuses. The left circumflex artery followed a periannular path, initially between the aortic wall and the left atrium, proceeding posteriorly between the aorta and the right pulmonary artery. We proceeded to aortic valve excision and annular debridement. Afterwards, a meticulous dissection of the anomalous circumflex artery was undertaken, starting from its origin, following to the lateral and posterior aspects of the aortic wall. To avoid kinking, both right and circumflex origins were buttoned from the aorta and, after complete dissection of the anomalous coronary (Figure 3), re-implanted at an higher level. Finally, a size 23 Perimount bioprosthesis was seated in place, under direct visualization of the anomalous coronary.

After coming off $\mathrm{CPB}$, the intraoperative transesophageal echocardiogram (TOE) revealed normal prosthesis function, preserved left ejection fraction and patent left circumflex artery (Figure 4). The post-operative course was uneventful, and the patient was discharged home after 6 days.

\section{Discussion}

Coronary artery anomalies occur in about 1-2\% of the general population but are more frequently described in association with bicuspid aortic valves. [1]

Anomalous origin of the circumflex artery from the right aortic sinus is a frequent variant and usually takes a retro artic and retro pulmonary route before reaching the left atrioventricular groove and is usually a benign finding, without any clinical consequence. [2] However, during aortic valve replacement, risks emerge, including (1) damage to the coronary during aortotomy or valve resection, (2) unseen coronary ostium obstruction by the prosthesis, (3) suture ligation of the artery during prosthesis implantation or (4) compression or distortion of the artery by the prosthesis ring. Complications may appear right after or soon after surgery, but also long time after, reportedly due to arrhythmogenic heart ischemia from circumflex artery compression and distortion over time. [3]

Although only a few reports addressed this issue to date, best outcomes are described when mobilization of the anomalous retro aortic coronary artery is performed. [4] Bypass grafting of the left circumflex has been used [5], but initial competitive flow from the patent native vessel may render it useless. Implantation of undersized prosthesis, with sutures low below the aortic annulus, did not avoid coronary compression and the need for urgent percutaneous intervention after surgery [5] .

Implantation of a stentless bioprosthesis may prevent the compressive effect of a rigid ring of the stented valves on the anomalous coronary.[2] However, the risk of coronary damage, distortion or ligation still exists, either during the excision of a calcified aortic valve and annular debridement or due to the close relation of the prosthesis sutures to the anomalous vessel, during valve implantation. Transcatheter aortic valve implantation may also compress a retro aortic left circumflex coronary artery, especially during valve balloon expansion.

\section{Conclusion}


We describe a case of an anomalous circumflex artery from the right aortic sinus, with retro aortic and justaannular course. Although benign, this common anatomic variant, particularly associated with bicuspid aortic valves, may cause immediate severe complications or sudden death, even years after aortic valve replacement. Exhaustive preoperative study, careful surgical planning and attention to the coronary origin and course are of uttermost importance when performing aortic valve surgery.

\section{References}

[1] Naito S, Petersen J, Reichenspurner H, Girdauskas E, The impact of coronary anomalies on the outcome in aortic valve surgery: comparison of bicuspid aortic valve versus tricuspid aortic valve morphotype. Interact CardioVasc Thorac Surg 2018;26:617-622.

[2] Shriki J, Shinbane J, Rashid M, Hindoyan A, Withey J, DeFrance Aet al , Identifying, Characterizing, and Classifying Congenital Anomalies of the Coronary Arteries. RadioGraphics 2012;32:453-468.

[3] Veinot JP, Acharya V, Bedard P, Compression of Anomalous Circumflex Coronary Artery by a Prosthetic Valve Ring. Ann Thorac Surg 2016;102:113-115

[4] Liebrich M, Tzanavaros I, Scheid M, Voth W, Doll KD, Hemmer W, Aortic valve/root procedures in patients with an anomalous left circumflex coronary artery and a bicuspid aortic valve: anatomical and technical implications. Interact CardioVasc Thorac Surg 2015;21:114-116.

[5] Alameddine A, Binnall B, Conlin F, Broderick P, Aortic Valve Replacement in 8 Adults with Anomalous Aortic Origin of Coronary Artery. Tex Heart Inst J 2019;46: 189-194

\section{Legends}

Figure 1 - Coronary angiogram frame showing an anomalous left circumflex artery arising very close to the right coronary artery and following a retro aortic course.

Figure 2 - Computed tomography angiogram of the thorax, showing the origin of the anomalous left circumflex artery (A), reaching its lowest point (B) before continuing between in the space between the aortic root and the left atrium $(\mathrm{C})$, and joining the usual path in the atrioventricular groove (D). Arrows point to the vessel in each frame.

Figure 3 - Circumflex artery freely dissected from the lateral and dorsal aortic walls.

Figure 4 - Doppler flow echocardiogram frame showing a patent vessel between the lower aorta and the left atrium.

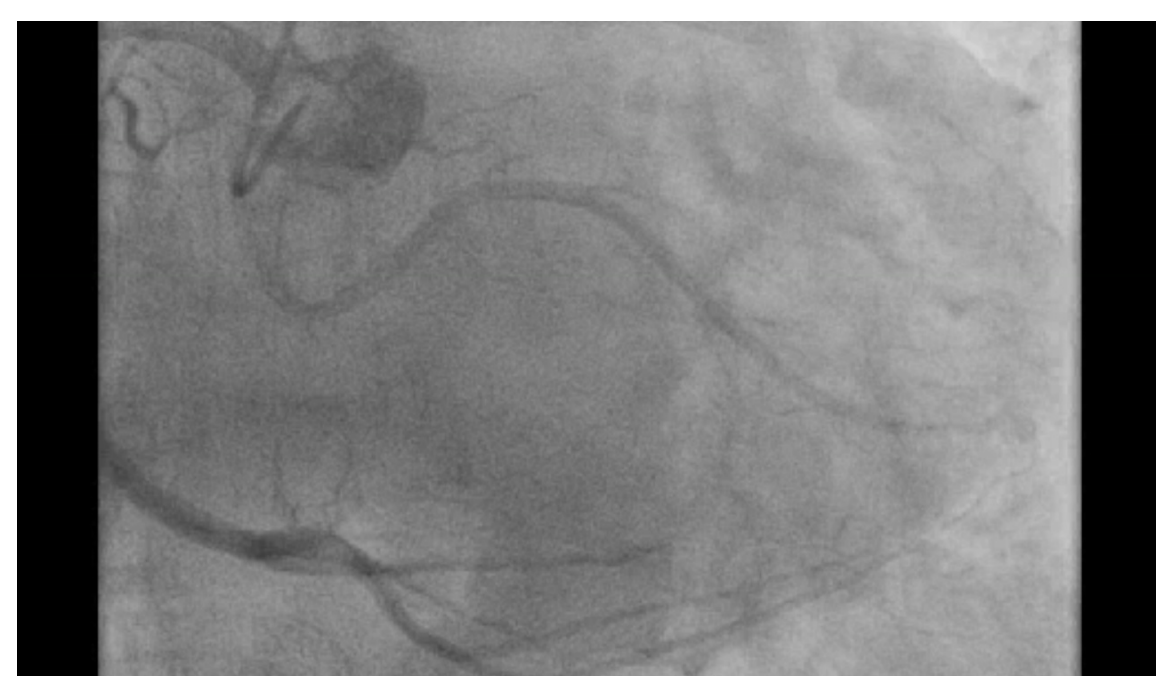



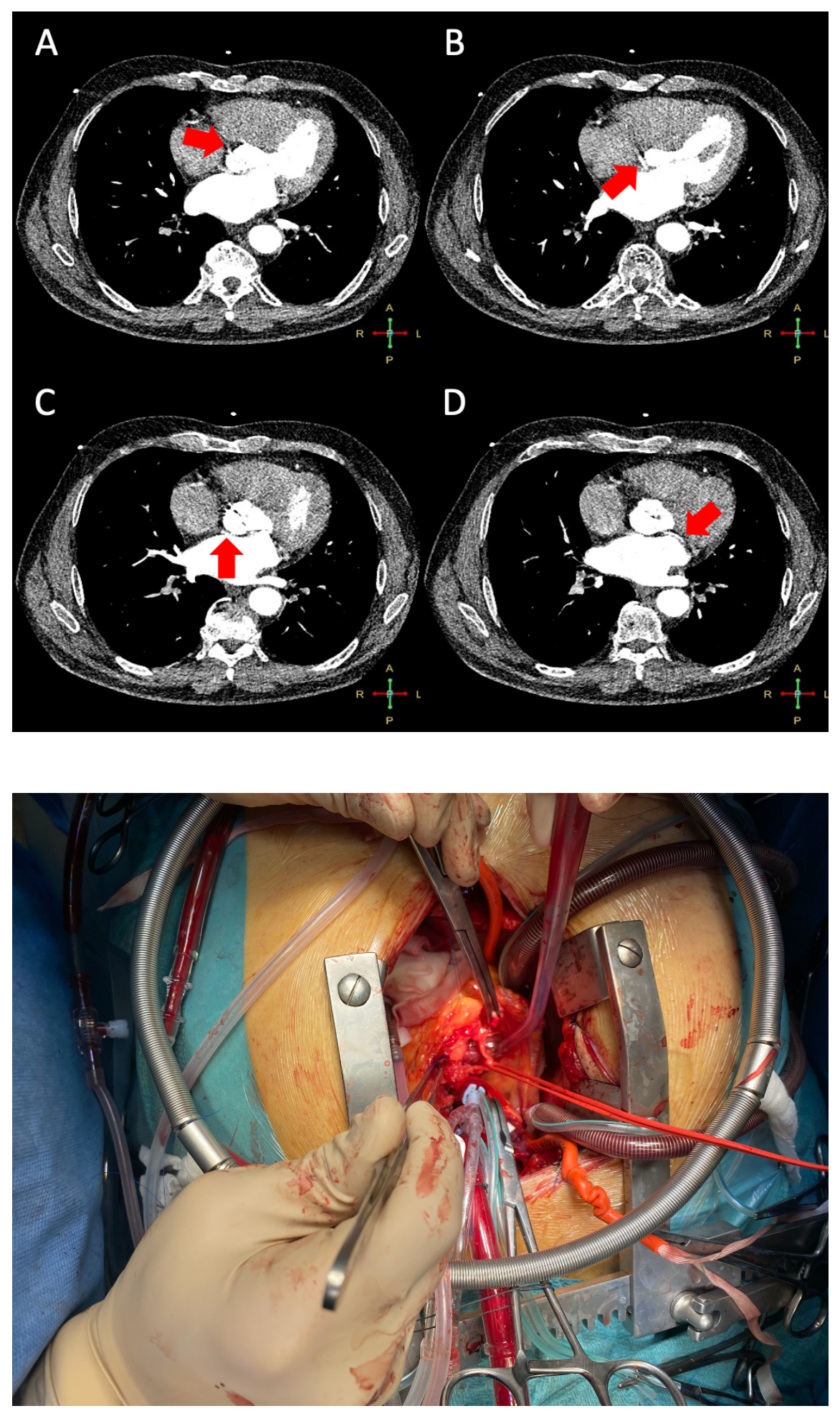


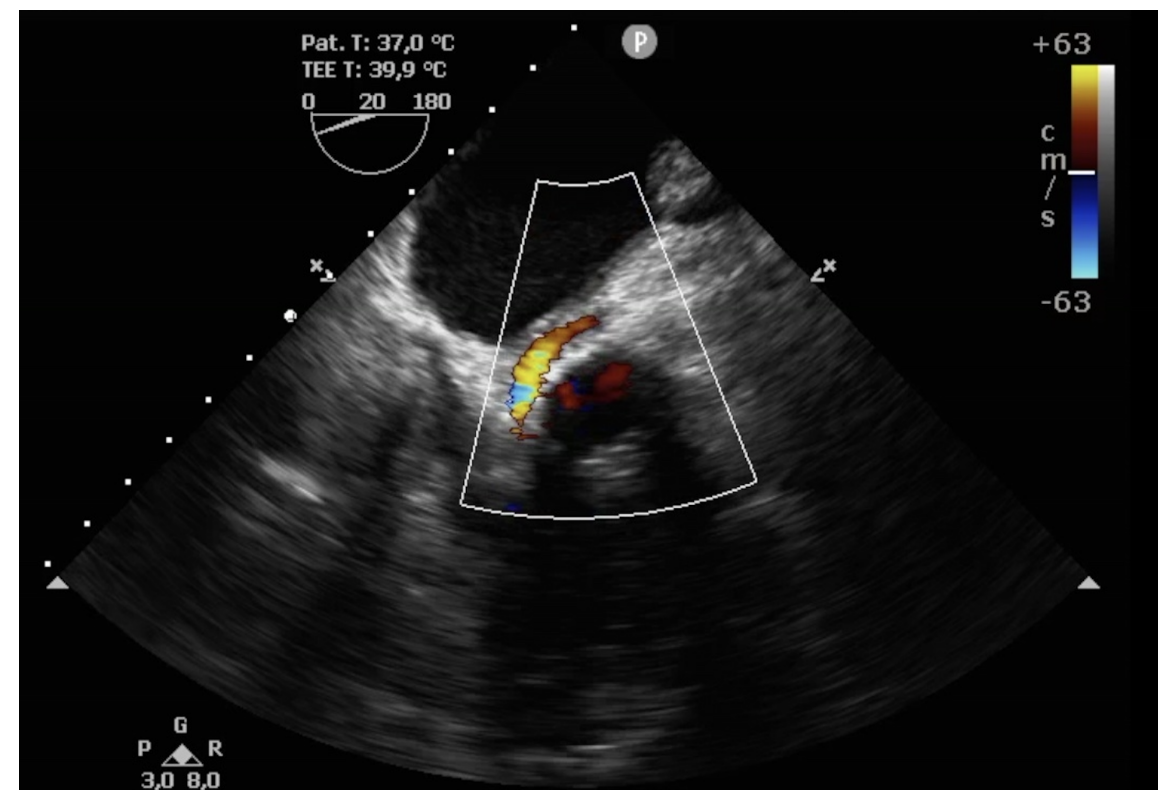

